\title{
Self-Assembly of Nanoporous Chiral Networks with Varying Symmetry from Sexiphenyl-dicarbonitrile on $\mathrm{Ag}(111)$
}

\author{
Dirk Kühne, ${ }^{*}, \dagger$ Florian Klappenberger, ${ }^{\dagger}$ Régis Decker, ${ }^{\ddagger}$ Uta Schlickum, ${ }^{\ddagger}$ Harald Brune, ${ }^{\ddagger}$ \\ Svetlana Klyatskaya, ${ }^{\S}$ Mario Ruben, ${ }^{\S}$ and Johannes V. Barth ${ }^{\dagger}$ \\ Physik Department E20, TU München, Germany, École Polytechnique Fédérale de Lausanne, Institut de \\ Physique de la Matière Condensée, CH-1015 Lausanne, Switzerland, and Institute of Nanotechnology, \\ Forschungszentrum Karlsruhe, Karlsruhe, Germany
}

Received: May 4, 2009; Revised Manuscript Received: August 31, 2009

\begin{abstract}
The self-assembly of sexiphenyl-dicarbonitrile molecules on the $\operatorname{Ag}(111)$ surface is investigated using lowtemperature scanning tunneling microscopy (STM) in ultrahigh vacuum. Several nanoporous networks with varying symmetry and pore size coexist on the surface after submonolayer deposition at room temperature. The different rectangular, rhombic, and kagomé shaped phases are commensurate with the $\mathrm{Ag}(111)$ substrate and extend over micrometer-sized domains separated by step edges. All phases are chiral and have very similar formation energetics. We attribute this to common construction principles: the approximately flatlying polyphenyl backbones following high-symmetry directions of the substrate, the epitaxial fit and the nodal motif composed of $\mathrm{CN}$ end groups laterally attracted by phenyl hydrogens. Close to saturation coverage, a single dense-packed phase prevails with all molecules aligned parallel within one domain. Our results demonstrate that porous networks of different complexity can evolve by the self-assembly of only one molecular species on a metal surface.
\end{abstract}

\section{Introduction}

Supramolecular engineering of porous molecular networks at well-defined surfaces emerged as a major research theme over the last years. ${ }^{1,2}$ Because of their tunable cavity size, they represent versatile functional nanoarchitectures. ${ }^{3}$ Thus, a series of bottom-up fabrication schemes ${ }^{4}$ has been developed to realize two-dimensional (2D) layers presenting well-defined open spaces, using hydrogen bonding, ${ }^{5-9}$ metal-directed assembly, ${ }^{10-14}$ the organization of flexible species, ${ }^{15-18}$ or covalent chemical reactions. ${ }^{19,20}$

Nanoporous networks featuring 2D chirality represent a chemically and topologically interesting subclass in this area. ${ }^{21-26}$ In earlier work, we employed simple ditopic dicarbonitrilepolyphenyl molecular building blocks and studied their selfassembly on the $\operatorname{Ag}(111)$ surface. ${ }^{27}$ Topologically different supramolecular structures formed depending on the backbone length. In particular, selecting species containing three, four, or five phenyl groups led to the formation of a close-packed chevron layer, a chiral open network with rhombic pores, or a nanoporous chiral kagomé network, respectively. All investigated networks of that study were reported to be commensurate with the underlying $\operatorname{Ag}(111)$ atomic lattice.

The employed carbonitrile functional group $(\mathrm{CN})$ as well as its anorganic cyanide counterpart $(\mathrm{CN}-)$ are well-known for organometallic complexation. ${ }^{28}$ The $\mathrm{CN}$ group is essential in conductive organic crystals ${ }^{29}$ and thin films $\mathrm{s}^{30}$ and plays a major role for metal-organic magnets ${ }^{31,32}$ recently reaching Curie temperatures above room temperature. ${ }^{33}$ In two dimensions, the bonding capabilities of two interacting $\mathrm{CN}$ groups have proven to steer the self-assembly of substituted porphyrins on $\mathrm{Au}(111){ }^{34}$

* To whom correspondence should be addressed. E-mail: dirk.kuehne@ mytum.de.

$\dagger$ TU München.

* Institut de Physique de la Matière Condensée.

${ }^{\S}$ Forschungszentrum Karlsruhe, Karlsruhe.
CHART 1: Sexiphenyl-dicarbonitrile

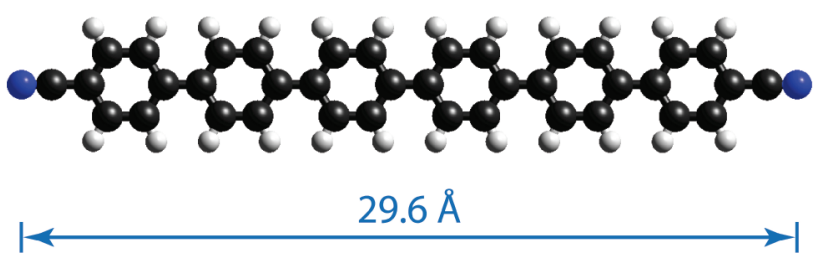

Depending on the position of the $\mathrm{CN}$ substituents, the formation of triangular clusters, tetramers, and supramolecular wires could be triggered. In a different work, the carbonitrile group controlled structure formation by interaction with alkoxy groups. ${ }^{35}$

Poly-para-phenyl backbones are furthermore interesting because solids or thin films made from this class of aromatic molecules provide a wide bandgap, ${ }^{36}$ making them specifically interesting for the construction of light emitting devices. ${ }^{37}$ Probably the most promising polyphenyl candidate is sexiphenyl, ${ }^{36,38,39}$ which combines blue photoluminescence ${ }^{36}$ with a high hole mobility. ${ }^{38}$ On the basis of $p$-sexiphenyl, organic thin film transistors have been fabricated. ${ }^{38}$ From an oriented $p$-sexiphenyl film, polarized electroluminiscence has been observed. ${ }^{40}$ Covering $p$-sexiphenyl with appropriate dye layers and filters can convert its deep blue emitted light into green and red, making full-color applications possible. ${ }^{41}$

In this work, we show that the related ditopic tecton, sexiphenyl-dicarbonitrile (Chart 1), self-assembles on $\operatorname{Ag}(111)$ into a multitude of highly regular, commensurate, and porous phases with varying symmetry. The achiral building block organizes into chiral networks appearing in both enantiomorphic forms. These various networks are restricted to submonolayer coverages. Approaching monolayer saturation, a single densepacked phase forms. The conjugated $\pi$-systems are uniformly oriented approximately parallel, face-on, with respect to the 


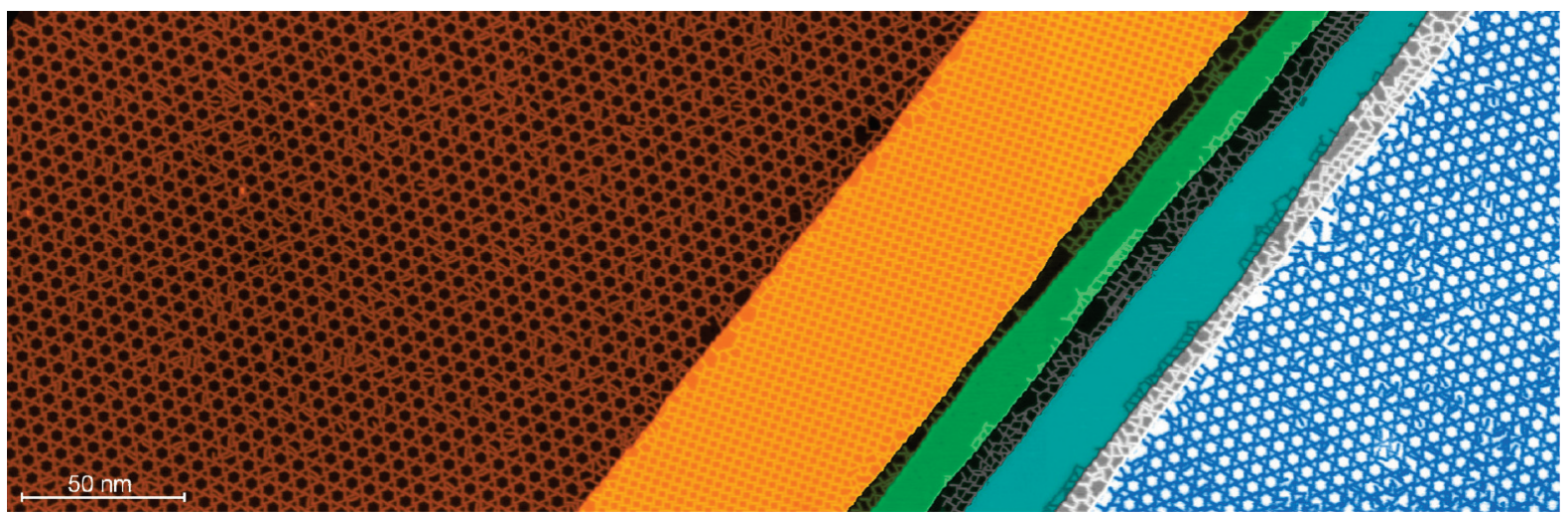

Figure 1. STM topography $\left(V_{\mathrm{B}}=1.25 \mathrm{~V}, I_{\mathrm{T}}=0.14 \mathrm{nA}\right)$ for $\sim 0.4 \mathrm{MLs}$ of sexiphenyl-dicarbonitrile molecules on $\mathrm{Ag}(111)$ deposited at $297 \mathrm{~K}$ and cooled down to $8 \mathrm{~K}$. On large terraces, the rectangular and kagomé network formed very regularly over wide areas. The phases are separated by step edges. Narrow terraces remain mainly uncovered or are filled by irregular networks.

substrate. In comparison to the growth of pure sexiphenyl, the functionalization with dicarbonitrile end groups improves the selectivity of the adsorption sites as well as the molecular alignment along surface high symmetry directions.

\section{Results and Discussion}

Supramolecular Chiral Phases. Figure 1 shows an exemplary large scale scanning tunneling microscopy (STM) topography after the deposition of $\sim 0.4$ monolayers (MLs) of sexiphenyl-dicarbonitrile molecules. One ML refers to one full dense-packed layer of molecules with their phenyl rings approximately parallel to the substrate. From the bottom right to the top left, there are eight terraces separated by descending monatomic steps. The sexiphenyl-dicarbonitrile molecules are imaged as rod-like protrusions with $\sim 27 \AA$ length and an apparent height of $1-1.5 \AA$ depending on bias and tip conditions. On more than $50 \mathrm{~nm}$ wide terraces and in the left part of Figure 1, kagomé networks (brown) and networks with rectangular pores (orange) form. The right-hand terrace in the STM topography shows a second kagomé network (blue), which is rotated by $30^{\circ}$ with respect to the one on the left-hand side. The coexistence of different regular networks is an indication for similar formation energies of these porous structures with different symmetry built by the same molecule. In between terraces covered with molecular networks, several other terraces can be found that remain undecorated (blue and green) apart from attachment to the lower side of the corresponding step edge. On narrow $(<20 \mathrm{~nm})$ terraces, irregular networks prevail presumably due to the influence of two step edge boundaries nearby.

In the deposition temperature range from 200 to $370 \mathrm{~K}$, no preferred phase has been found. This shows all of the different phases have similar formation energies. After deposition at a substrate temperature of $110 \mathrm{~K}$ and cooling down for STM imaging, no regular network has been found (see data provided in the Supporting Information). Therefore, the networks are formed by two-dimensional self-assembly, requiring substantial molecular mobility, and the various ordered structures are driven by the formation of weak links between adjacent molecules.

Three different kinds of regular structures have been predominantly observed for submonolayer coverages: a rectangular (Figure 2a and b), a kagomé (Figure 2c and d), and a rhombic phase (Figure 2e and $\mathrm{f}$ ). All three networks have nodal binding motifs where the ends of four molecules join together. Attraction results from $\mathrm{H} \cdots \mathrm{N}$ bonds in between the $\mathrm{CN}$ end groups of the molecules and the hydrogens of the aromatic rings of an adjacent molecule and from dipole-dipole interaction introduced by the $\mathrm{CN}$ groups. ${ }^{27,34,42}$ Molecules in the rhombic phase oriented from the top left to the bottom right show a brighter contrast than molecules oriented from the top right to the bottom left (Figure 2e), which can be attributed to different adsorption sites. Similarly, a highly regular pattern of different brightnesses is reported for the rhombic network of $\mathrm{NC}-\mathrm{Ph}_{4}-\mathrm{CN}$ molecules. ${ }^{27}$ Details on the bonding properties and differences between the distinct phases will be discussed in the section Substrate Epitaxy.

All nodal motifs, and thus the networks based on them, exhibit chirality, originating from the supramolecular organization of achiral molecules on an achiral surface. ${ }^{43}$ In the structures shown in the left column of Figure 2, the nodes are oriented counterclockwise, whereas, in the right column, they are oriented clockwise. Our data indicates the molecules have an equal probability to assemble into the one or other enantiomorphic arrangement for all three phases. The kagomé domain exhibits $P_{6}$ symmetry.

Rarely, a mixed rhombic phase (Figure $2 \mathrm{~g}$ ) appears consisting of similar 4-fold nodes as the kagomé network unifying both chiral motifs and therefore forming a racemic mixture: Along one horizontal line (dashed line), nodes are rotating clockwise, and in the adjacent horizontal row (dotted line), nodes are rotating counter-clockwise. This network features point chirality. The obvious 50:50 ratio of the two mirror forms clearly corroborates that both nodes are equivalent in energy.

In an earlier study ${ }^{24}$ on the self-assembly of 4-[trans-2-(pyrid4-yl-vinyl)]benzoic acid (PVBA) on $\mathrm{Cu}(100)$, regular networks have been found in which a 4-fold node appeared similar to the node in the rectangular network reported here. In the case of PVBA/Cu(100), the molecules and the nodes feature 2D chirality. Each enantiomer of the PVBA node is made purely from one of the PVBA enantiomers. In our case, all chiral properties originate from organization of one achiral molecular species only. Organizational chirality has also been observed for $\mathrm{NC}-\mathrm{Ph}_{4}-\mathrm{CN}$ and $\mathrm{NC}-\mathrm{Ph}_{5}-\mathrm{CN}$ on $\mathrm{Ag}(111){ }^{27}$

For coverages close to ML saturation, a dense-packed phase (Figure 3) prevails. This phase can be assembled and keeps stable at room temperature. It does not exhibit a 4-fold node: Within one domain, all molecules align parallel and form molecular nanostripes. The stripe direction is at $84.3 \pm 0.5^{\circ}$ to the long axes of the molecules. Within a nanostripe, the periodicity is $7.4 \AA$. Two neighboring molecular nanostripes are displaced half the stripe periodicity laterally along the stripe direction and separated 27.2 $\AA$ parallel to the long molecular 

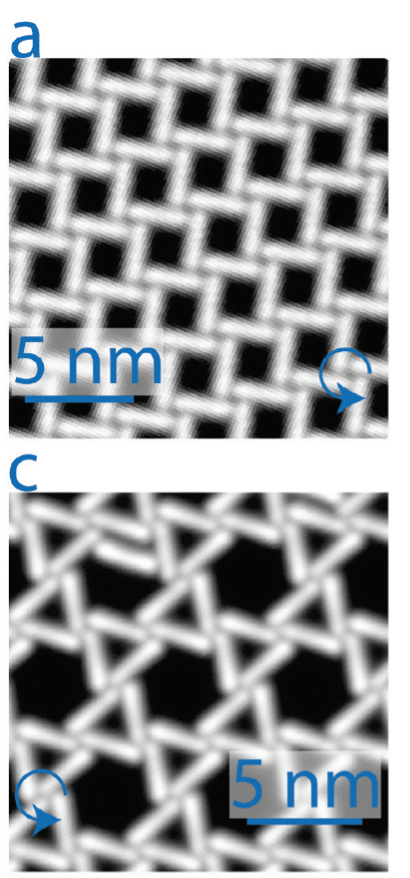

e

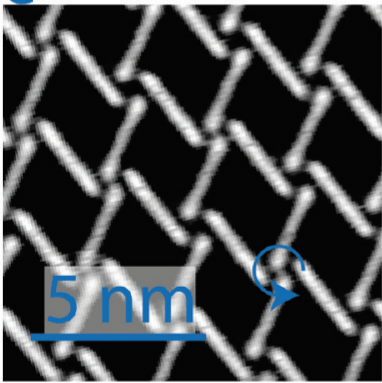

g

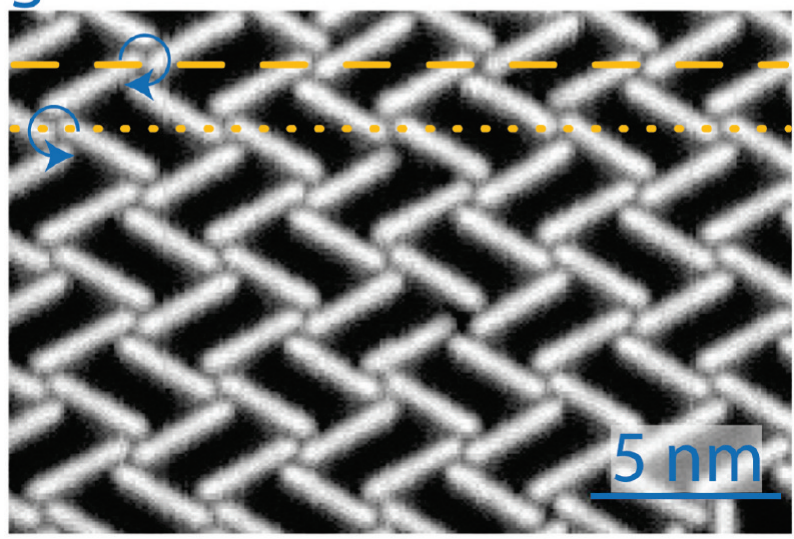

Figure 2. STM topographies of different regular networks coexisting at coverages less than 0.5 MLs. (a) Rectangular network $\left(V_{\mathrm{B}}=1.90\right.$ $\left.\mathrm{V}, I_{\mathrm{T}}=0.1 \mathrm{nA}\right)$. (b) Mirror symmetric rectangular network $\left(V_{\mathrm{B}}=0.55\right.$ $\left.\mathrm{V}, I_{\mathrm{T}}=0.1 \mathrm{nA}\right)$. (c) Kagomé network $\left(V_{\mathrm{B}}=-0.5 \mathrm{~V}, I_{\mathrm{T}}=0.1 \mathrm{nA}\right)$. (d) Mirror symmetric kagomé network $\left(V_{\mathrm{B}}=0.9 \mathrm{~V}, I_{\mathrm{T}}=0.13 \mathrm{nA}\right)$. (e) Rhombic network $\left(V_{\mathrm{B}}=0.65 \mathrm{~V}, I_{\mathrm{T}}=0.05 \mathrm{nA}\right)$. (f) Mirror symmetric rhombic network $\left(V_{\mathrm{B}}=0.7 \mathrm{~V}, I_{\mathrm{T}}=0.05 \mathrm{nA}\right)$. $(\mathrm{g})$ Mixed phase incorporating both enantiomorphic forms of the nodes $\left(V_{\mathrm{B}}=3.13 \mathrm{~V}\right.$, $\left.I_{\mathrm{T}}=0.14 \mathrm{nA}\right)$.

axes. This separation is smaller than the length of the molecule and smaller than the $29.5 \AA$ found for $p$-sexiphenyl on $\mathrm{Ag}(111) .{ }^{44}$ Therefore, we conclude that the carbonitrile groups of adjacent stripes interdigitate (see model in the section Substrate Epitaxy). When more molecules are added, they do not edge into the dense-packed molecular monolayer but form

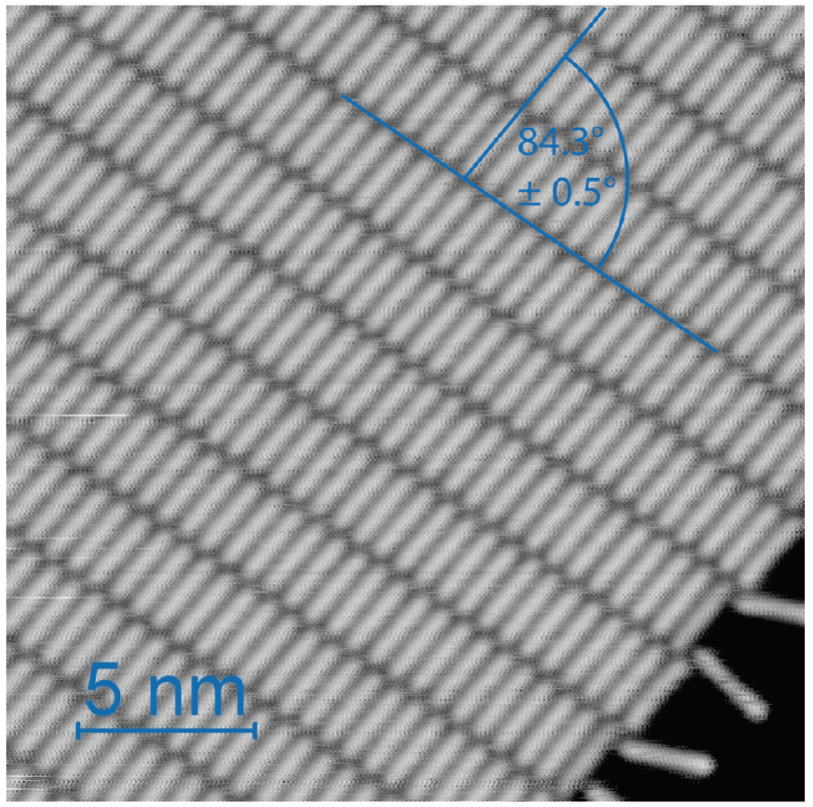

Figure 3. STM topography of the dense-packed phase prevailing at coverages close to ML saturation $\left(V_{\mathrm{B}}=-1.22 \mathrm{~V}, I_{\mathrm{T}}=0.08 \mathrm{nA}\right)$.

a second layer on top. The molecules have their phenyl rings parallel, face-on, to the substrate surface. This is different from the case of $p$-sexiphenyl added onto a face-on $p$-sexiphenyl ML where the extra molecules align edge-on with all of their phenyl rings oriented approximately perpendicular to the $\operatorname{Ag}(111)$ surface. ${ }^{44}$

Substrate Epitaxy. In this section, the epitaxy of the different networks with respect to the substrate will be discussed. Molecules and the underlying atomic corrugation have been resolved simultaneously (Figure 4a). Such favorable imaging conditions occur rarely. Most likely, they are due to a tip to that some molecules have been transferred. ${ }^{45}$ The data evidence that in the rectangular phase molecules are aligned in the crystallographic $\langle\overline{1} 10\rangle$ and $\langle\overline{1} 12\rangle$ directions. With a different tip state with good lateral resolution for intramolecular features, the molecules oriented in the two directions are imaged differently (Figure 4b). Whereas the molecules in the [110] direction appear with a zigzag contour, the ones in the [112] direction show a shape symmetric to their long molecular axis. A zigzag contour similar to the one we found here has been reported for sexiphenyl on $\operatorname{Ag}(111) .^{45,46}$ There, the authors showed that the edge of a phenyl ring appears bright if the center of the corresponding off-axis $\mathrm{C}-\mathrm{C}$ bond is located on top of a silver surface atom; if the center of a $\mathrm{C}-\mathrm{C}$ bond is positioned over a bridge site, the corresponding phenyl ring edge appears darker (Figure 4c). The similar alignment of sexiphenyl and $\mathrm{NC}-\mathrm{Ph}_{6}-\mathrm{CN}$ in $\langle 110\rangle$ implies that the aromatic backbone plays an important role for the epitaxy. The appearance of the zigzag contour and the analysis of Figure 4 a consistently indicate the epitaxial position of the molecules aligned in $\langle\overline{1} 10\rangle$, as depicted in Figure $4 \mathrm{c}$, thus providing a good starting point for the construction of a structure model.

Keeping in mind the epitaxial orientations of the rectangular phase, the orientations of the three other phases have been determined. The structure models for all four phases are collectively presented in Figure 5. In each panel, the atoms (gray circles) and the ball-and-stick models of the molecules are superimposed on the experimental data, in which the molecules appear as white regions. The unit cells are indicated by the transparent blue regions. 
a

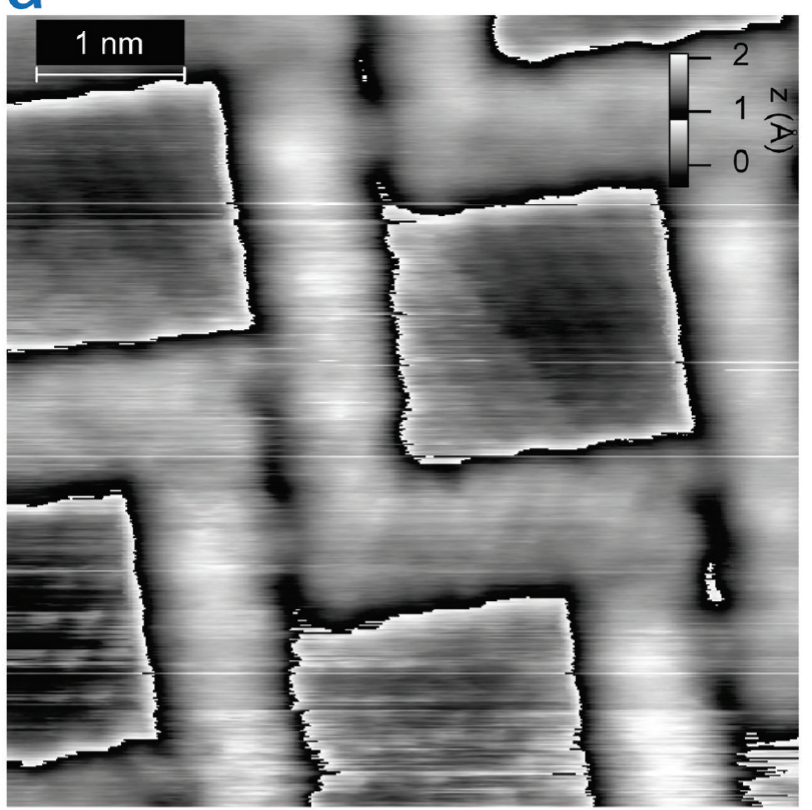

b
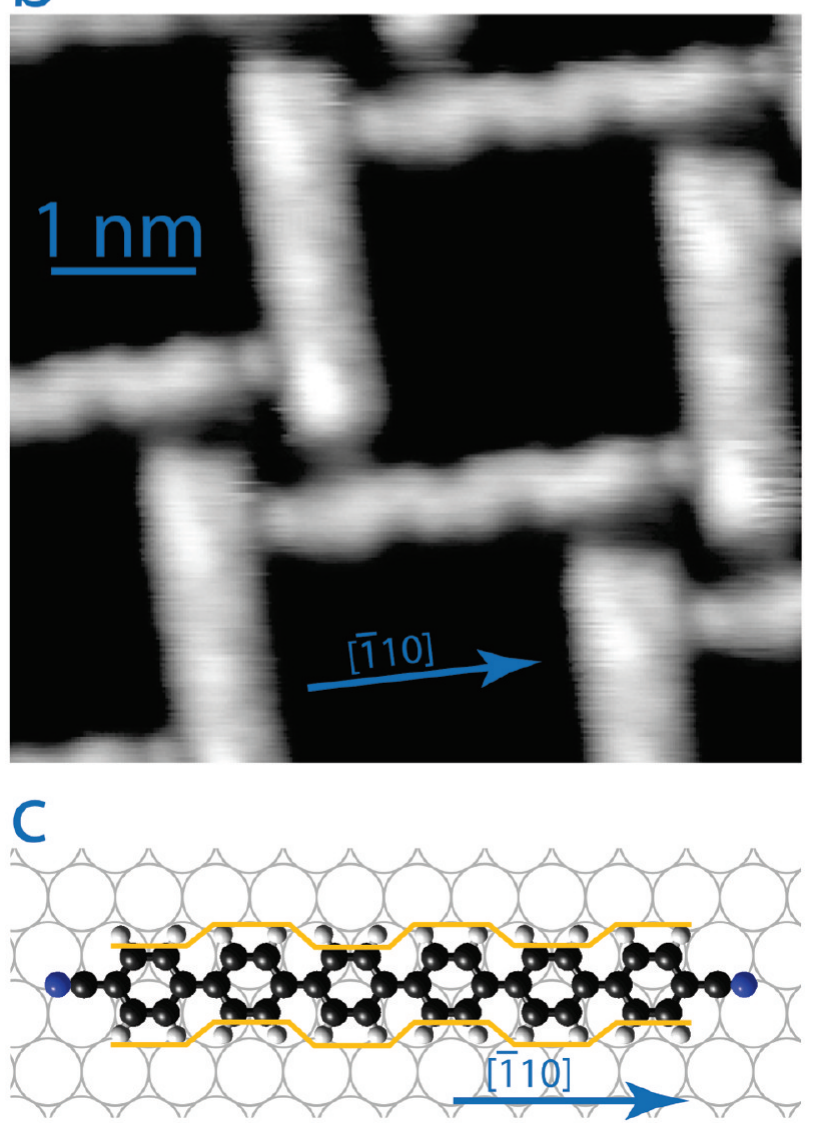

Figure 4. (a) STM topography of the rectangular phase recorded with unusual imaging conditions where the underlying atomic lattice appears together with the molecules. The data confirms the orientation of the rectangular network with respect to the substrate (a repetitive color scale is used here to visualize both atoms and molecules better). ( $\left.V_{\mathrm{B}}=-1.15 \mathrm{~V}, I_{\mathrm{T}}=0.1 \mathrm{nA}\right)$. (b) An image obtained with a tip featuring high lateral resolution. Molecules oriented along the $[110]$ direction exhibit a zigzag pattern $\left(V_{\mathrm{B}}=\right.$ $0.55 \mathrm{~V}, I_{\mathrm{T}}=0.1 \mathrm{nA}$ ). (c) Model. Position of a zigzag patterned molecule with respect to the substrate. The zigzag curvature is indicated by the solid orange line.
In three dimensions, para-polyphenyls typically rotate adjacent phenyl rings because of repulsion between hydrogen atoms of adjacent phenyl rings, but the long molecular axis stays straight. ${ }^{47-49}$ The effect of such rotations on our epitaxy models is only minor. Thus, for simplicity, we depict planar molecules in our models. Their length is expected to be close to the one in the gas phase that is a $2.96 \mathrm{~nm}$ distance from nitrogen to nitrogen atom of their carbonitrile end groups. The observed structures do not show a Moiré pattern for all tunneling conditions we tested; therefore, commensurate models have been constructed. For comparison, the noncommensurate supercell of tetrapyridyl-porphyrin on $\mathrm{Ag}(111)$ resulted in an evident modulation of the apparent height of the molecules independent of bias voltage, ${ }^{50}$ whereas the apparent height of our molecules is identical for adjacent unit cells.

In the rectangular network (Figure 5a), adjacent molecules include an angle of $90^{\circ}$. Distances have been determined between the long axes of the molecules. The bigger rectangular pore counts 10 atomic nearest neighbor distances in the [110] direction (labeled $\vec{a}$ ) and 11 atomic row distances in the orthogonal direction. The smaller pore inside the node adds up to 3.5 atomic nearest neighbor distances in the $\vec{a}$ direction and 4 atomic row distances in the orthogonal direction. The nodes consist of four molecules and are point symmetric with respect to their center. The density of the rectangular network is one molecule per 62 rhombic unit cells of the $\operatorname{Ag}(111)$ surface. The unit cell of the rectangular network is given by

$$
\left(\begin{array}{l}
\vec{c}_{\mathrm{re}} \\
\vec{d}_{\mathrm{re}}
\end{array}\right)=\left(\begin{array}{ll}
12 & -4 \\
-2 & 11
\end{array}\right)\left(\begin{array}{l}
\vec{a} \\
\vec{b}
\end{array}\right)
$$

where $\vec{a}$ and $\vec{b}$ are the $\operatorname{Ag}(111)$ unit vectors. Two molecules are in one unit cell of the rectangular network. The $\mathrm{H} \cdots \mathrm{N}$ bond lengths vary from 1.8 to $2.7 \AA$. Rectangular domains rotated $30^{\circ}$ with respect to each other have been observed. This can be rationalized because the structure in Figure 5a is invariant upon a rotation of the underlying $\operatorname{Ag}(111)$ lattice by $30^{\circ}$.

The density of the kagomé network is one molecule per 71.17 unit cells of the $\operatorname{Ag}(111)$ surface. Adjacent molecules in this network include angles of 0,60 , or $120^{\circ}$ (Figure 5b). In the kagomé network, there are two different pore shapes and sizes. The bigger pore has a quasi-hexagonal shape, and the smaller one features a triangle. Each quasi-hexagon is surrounded by six triangles, and each triangle forms part of the sidewalls of three different quasi-hexagons. The unit cell of the kagomé network is given by

$$
\left(\begin{array}{l}
\vec{c}_{\mathrm{ka}} \\
\vec{d}_{\mathrm{ka}}
\end{array}\right)=\left(\begin{array}{ll}
23 & -6 \\
6 & 17
\end{array}\right)\left(\begin{array}{l}
\vec{a} \\
\vec{b}
\end{array}\right)
$$

Six molecules are in one unit cell of the kagomé network, and the projected $\mathrm{H} \cdots \mathrm{N}$ bond lengths vary from 2.3 to $3.2 \AA$. These lengths are longer than $1.5 \AA$ reported for the kagomé lattice of $\mathrm{NC}-\mathrm{Ph}_{5}-\mathrm{CN}$ molecules. ${ }^{27}$ The $\mathrm{NC}-\mathrm{Ph}_{5}-\mathrm{CN}$ kagomé accommodates two brighter and two times two darker nodes, each two diagonally arranged. This kind of contrast difference has not been recorded for the $\mathrm{NC}-\mathrm{Ph}_{6}-\mathrm{CN}$ kagomé, indicating a single type of node. We believe the longer intermolecular distances within the $\mathrm{NC}-\mathrm{Ph}_{6}-\mathrm{CN}$ kagomé allow the right and left ends of the molecules to be more planar than in the case of the $\mathrm{NC}-\mathrm{Ph}_{5}-\mathrm{CN}$ kagomé. 
a

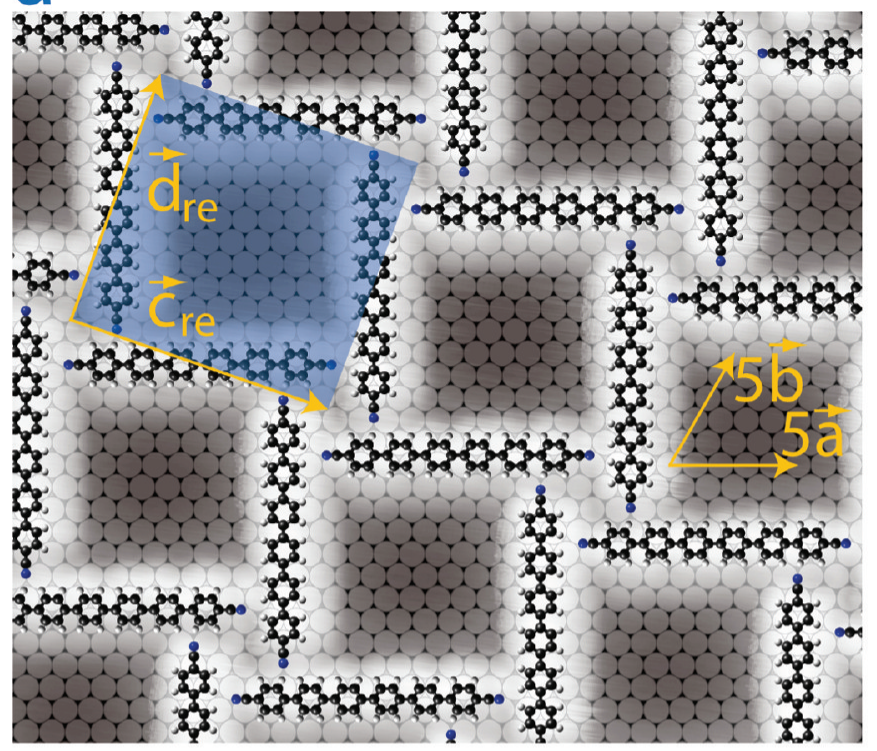

C

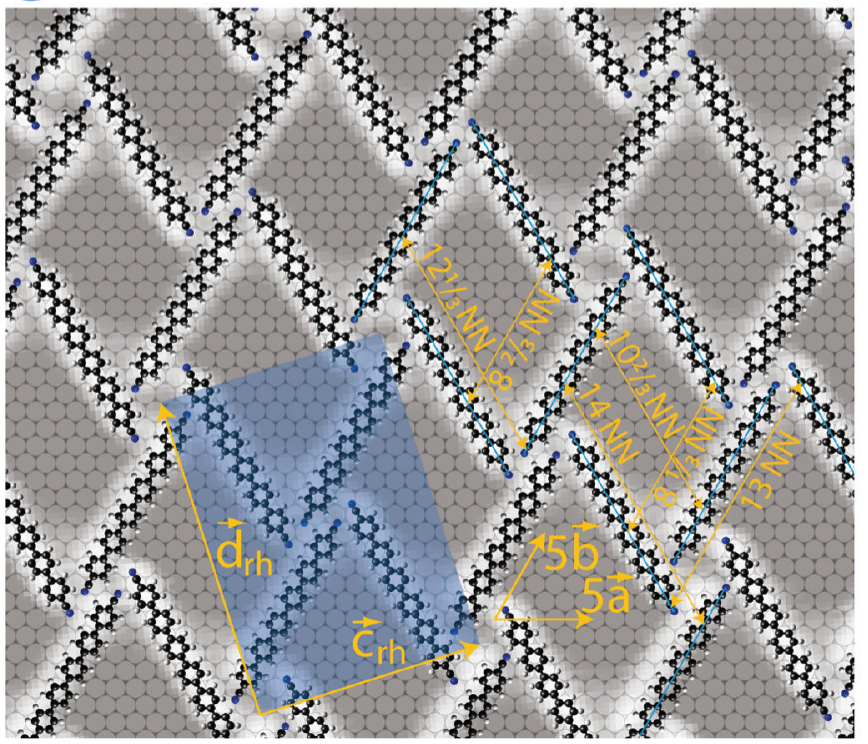

b
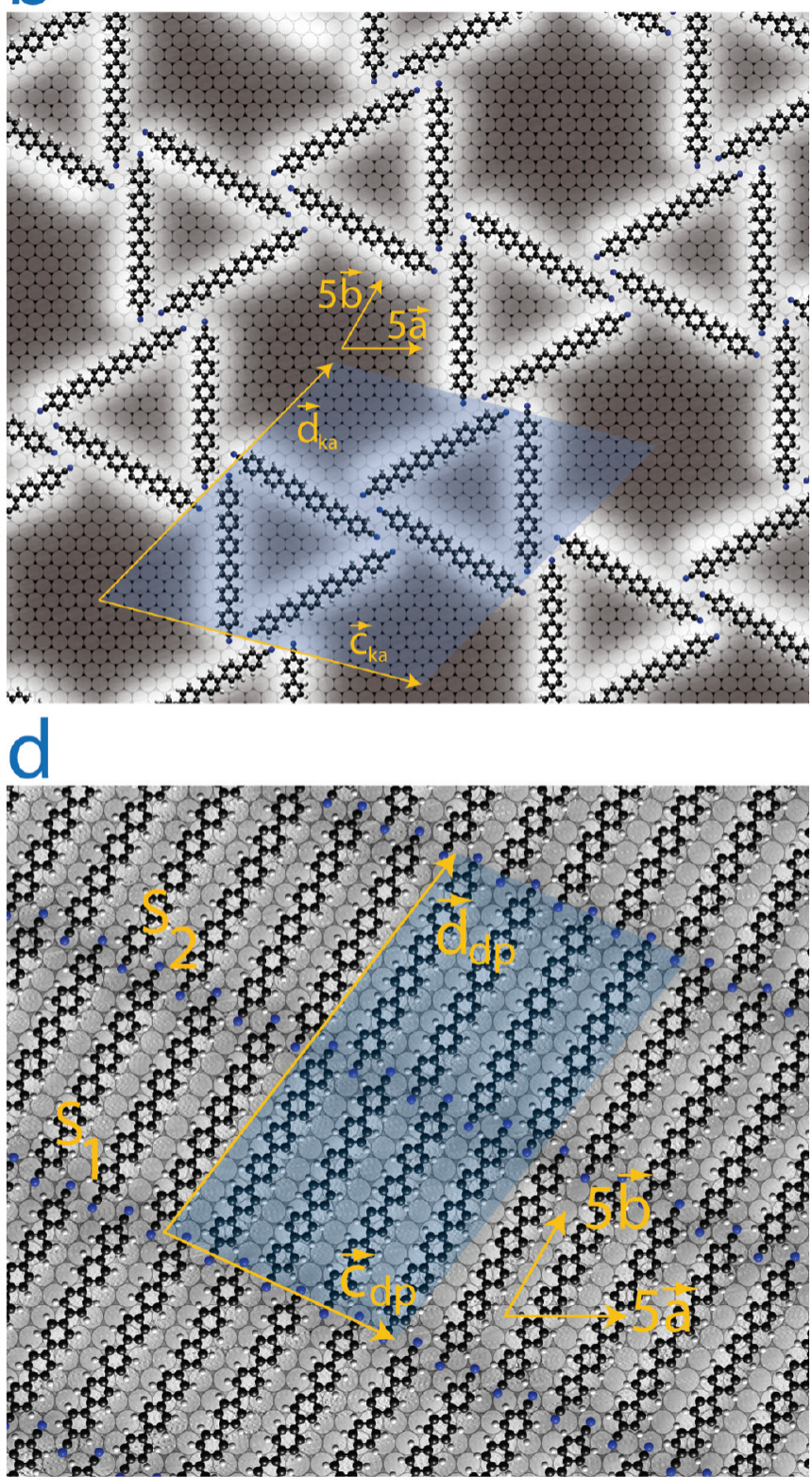

Figure 5. Ball-and-stick models: (a) rectangular network; (b) kagomé network; (c) rhombic network (NN: atomic nearest neighbor distance); (d) dense-packed network.

The structure model for the rhombic network is shown in Figure 5c. Distances are denoted between the long axes of the molecules. They give spacings within the nodes and the rhombi. In one row of rhombi in the $\vec{c}_{\text {rh }}$ direction, rhombi are bigger, and in an adjacent row, they are smaller. The unit cell of the rhombic phase can be expressed by

$$
\left(\begin{array}{l}
\vec{c}_{\mathrm{rh}} \\
\vec{d}_{\mathrm{rh}}
\end{array}\right)=\left(\begin{array}{ll}
9 & 4 \\
-14 & 18
\end{array}\right)\left(\begin{array}{l}
\vec{a} \\
\vec{b}
\end{array}\right)
$$

and contains four molecules. In this model, every second molecule points from the top left to the bottom right. These molecules had to be shifted away from the position where their $\mathrm{CN}$ end groups sit above the hollow sites of the $\mathrm{Ag}(111)$ surface by the sixth part of an atomic row distance to obtain agreement with the measured data. The projected $\mathrm{H} \cdots \mathrm{N}$ bond lengths range between 1.6 and $3.1 \AA$. The short bond length has been determined assuming a planar orientation of the corresponding phenyl ring and must be excluded in view of the interaction potentials. Similar to the $\mathrm{NC}-\mathrm{Ph}_{5}-\mathrm{CN}$ kagomé, ${ }^{27}$ it is very likely that tilted phenyl ring conformations allow such a dense packing.

The dense-packed phase is shown in Figure $5 \mathrm{~d}$ and defines a ML of sexiphenyl-dicarbonitrile molecules on $\operatorname{Ag}(111)$. There is one molecule in $2.09 \mathrm{~nm}^{2}$ in this tight assembly of molecules. The unit cell can be expressed by

$$
\left(\begin{array}{l}
\vec{c}_{\mathrm{dp}} \\
\vec{d}_{\mathrm{dp}}
\end{array}\right)=\left(\begin{array}{cc}
12 & -5 \\
3 & 18
\end{array}\right)\left(\begin{array}{l}
\vec{a} \\
\vec{b}
\end{array}\right)
$$

and contains eight molecules. The $\mathrm{H} \cdot \cdots \mathrm{N}$ bond length is $1.9 \AA$ for planar molecules in our model. The molecular distance of $7.54 \AA$ in the $\vec{c}_{\mathrm{dp}}$ direction is in good agreement with the experimental value of $7.4 \AA$ observed in line profiles that have 

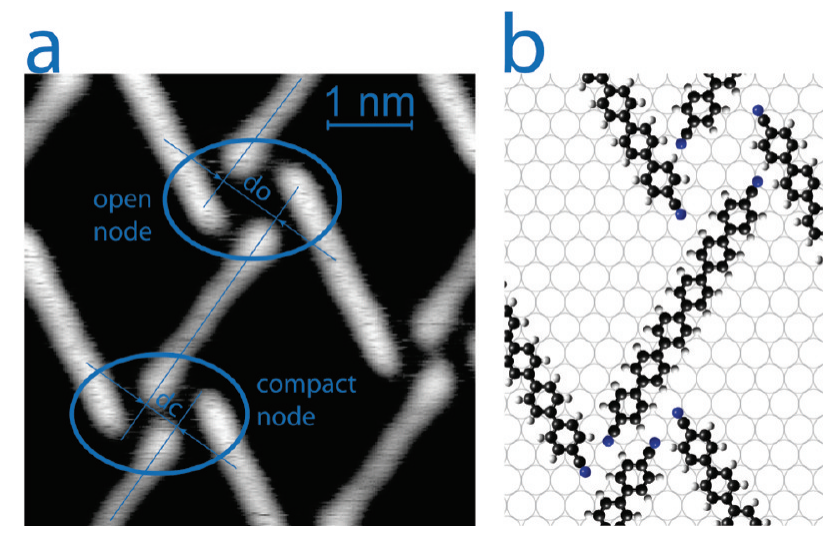

Figure 6. (a) High-resolution STM data exemplifying the two different binding motifs, the open and the compact node, within the rhombic network. The molecular separation $d o$ in the open node is 2 times the separation $d c$ in the compact node $\left(V_{\mathrm{B}}=0.65 \mathrm{~V}, I_{\mathrm{T}}=0.05 \mathrm{nA}\right)$. (b) Model.

been taken through molecular stripes (cf. Figure 3). A somewhat smaller axis to axis distance of $7 \AA$ for neighboring molecules within a molecular stripe has been reported for a phase of $p$-sexiphenyl molecules with their phenyl rings lying parallel to the $\operatorname{Ag}(111)$ substrate. ${ }^{44}$

In the model of the dense-packed phase (Figure 5d), two kinds of molecule nanostripes alternate. They are separated by $9 \frac{5}{8}$ atomic nearest neighbor distances in the [110] direction, in which the molecules are aligned with their long molecular axes and which is at $84.5^{\circ}$ to the molecular stripes. In stripe $S_{1}$ (Figure $5 \mathrm{~d})$, the long axis of the molecules is centered between two atomic rows. $\mathrm{CN}$ end groups are positioned above bridge or hollow sites, similar to end group positions in all three open porous networks. In stripe $S_{2}$ (Figure $5 d$ ), the molecules adopt positions where they center phenyl rings over on top positions. This kind of position has already been found for sexiphenyl molecules on $\mathrm{Au}(111)$, where they show the tendency to adopt orientations centering as many phenyl rings as possible over substrate atom centers. ${ }^{51}$ In both stripes of the dense-packed phase, molecular adsorption sites repeat every fourth $\mathrm{NC}-\mathrm{Ph}_{6}-\mathrm{CN}$ molecule along the stripe direction. Thus, the unit cell contains four molecules in each kind of nanostripe. The slightly different positions of the four molecules do not lead to a difference in apparent height. Similarly, no long-range height modulation is reported for a monolayer of pure sexiphenyl on $\operatorname{Ag}(111) .^{44}$

For $p$-sexiphenyl deposited onto oxidized silicon substrates, molecular ordering has been reported to be improved at elevated temperatures compared to room temperature deposition, ${ }^{38}$ similar to $p$-sexiphenyl on $\operatorname{Ag}(111)$ where annealing subsequent to deposition led to well-ordered $p$-sexiphenyl nanostripes. ${ }^{44}$ For the dense-packed phase presented here, annealing after room temperature deposition is not required to achieve perfect molecular ordering.

As displayed in the high resolution data (Figure 6a), two types of nodes are present in the rhombic network: open and compact ones with a measured distance ratio of $d_{\mathrm{o}} / d_{\mathrm{c}}=2$. To comply with this distance ratio, molecules are shifted away from the middle of two atomic rows in the model (Figure 6b). This leads to noninteger lengths in multiples of atomic nearest neighbor distances (NN) for the boundaries of the rhombi (Figure 5c). Accordingly, the model depicts two types of nodes: the compact with the distance $d_{\mathrm{c}}$ of $5 / 3$ atomic row distances and the open node with the distance $d_{\mathrm{o}}$ being twice as much. The compact node of the rhombi is similar to the node of the kagomé network, which is compact, too. In both arrangements, parallel end groups of adjacent molecules are very close and therefore dipole-dipole interaction by the $\mathrm{CN}$ groups might be noticeable. ${ }^{52}$

The distribution of open and compact nodes within two different domains of the rhombic phase has been analyzed and encoded in a schematic representation (Figure 7). In the vicinity of a step edge, the nodes are distributed regularly and straight rows of open and compact nodes next to each other starting at the step edge and continuing into the terrace alternate. Going further toward the interior of a terrace, the node distribution exhibits a uniform intermediate region (highlighted with a red boundary) that is equal for both domains in Figure 7. Having passed that intermediate region, an area is found dominated by the compact nodes. A detailed understanding of the reasons responsible for the node distribution cannot be provided. This example shows how manifold structure formation can be even for the case of a single tecton on a regular surface.

Compared to the dense-packed phase, the open porous networks are roughly 2 times less dense (Table 1). The kagomé has the smallest density, the rectangular network is more dense, and the rhombic phase has the highest density of all open porous networks. However, these densities differ from each other merely by $\sim 0.1$ MLs. For pentacene on $\mathrm{Au}(111)$, many ordered $2 \mathrm{D}$ structures have been found coexisting on different areas of the surface corresponding to slightly different coverages. ${ }^{53}$ If the free energy per unit area is a function of the molecule concentration, ${ }^{54}$ the densities of the kagomé, rectangular, and rhombic network should represent local minima of this function. Because the concentrations of these three phases are so close, also the three local minima are, and consequently they are not separated by high energy barriers. Therefore, during selfassembly, slight local variations in molecule density could steer the assembly to the one or other minimum.

In the kagomé network, a guest, such as an additional sexiphenyl-dicarbonitrile molecule, can stay inside a quasihexagonal pore. No guests have been found in the rectangular or rhombic networks. Only the structure with the lowest density houses an additional molecule. If a sexiphenyl-dicarbonitrile molecule is added into each quasi-hexagon of the kagomé phase, the density of this new structure exceeds the one of the rectangular phase and is still smaller than the density of the rhombic phase: 0.473 times a ML. Thus, the mechanism that allows picking up guests can lead to an overall equilibration of network densities.

In the rhombic phase, molecules oriented in our models from the top left to the bottom right are moved out of the middle of two atomic rows by the third part of an atomic row distance and molecules pointing from the top right to the bottom left by the sixth part. Every second molecule in the dense-packed phase centers phenyl rings over substrate surface atoms. This effect of occupying new adsorption sites becomes more prominent the more molecules per unit area phases have. We suggest that the stronger molecule-molecule interaction within the dense-packed phase leads to molecular positions with respect to the substrate that have not been observed for open porous networks.

For the models, commensurability of the networks is assumed and the molecules lie along the $\langle\overline{1} 10\rangle$ and $\langle\overline{1} 12\rangle$ directions of the $\operatorname{Ag}(111)$ surface. Nodes are constructed point symmetric. It turns out that for the porous networks the position of the center of the $\mathrm{CN}$ group is always near the hollow or bridge sites of the $\operatorname{Ag}(111)$ surface. Theoretical calculations for benzonitrile molecules adsorbed on the $\mathrm{Au}(111)$ surface have been reported in ref 42. This ab initio study has shown that monomer, dimer, and trimer structures preferentially arrange with the cyano 

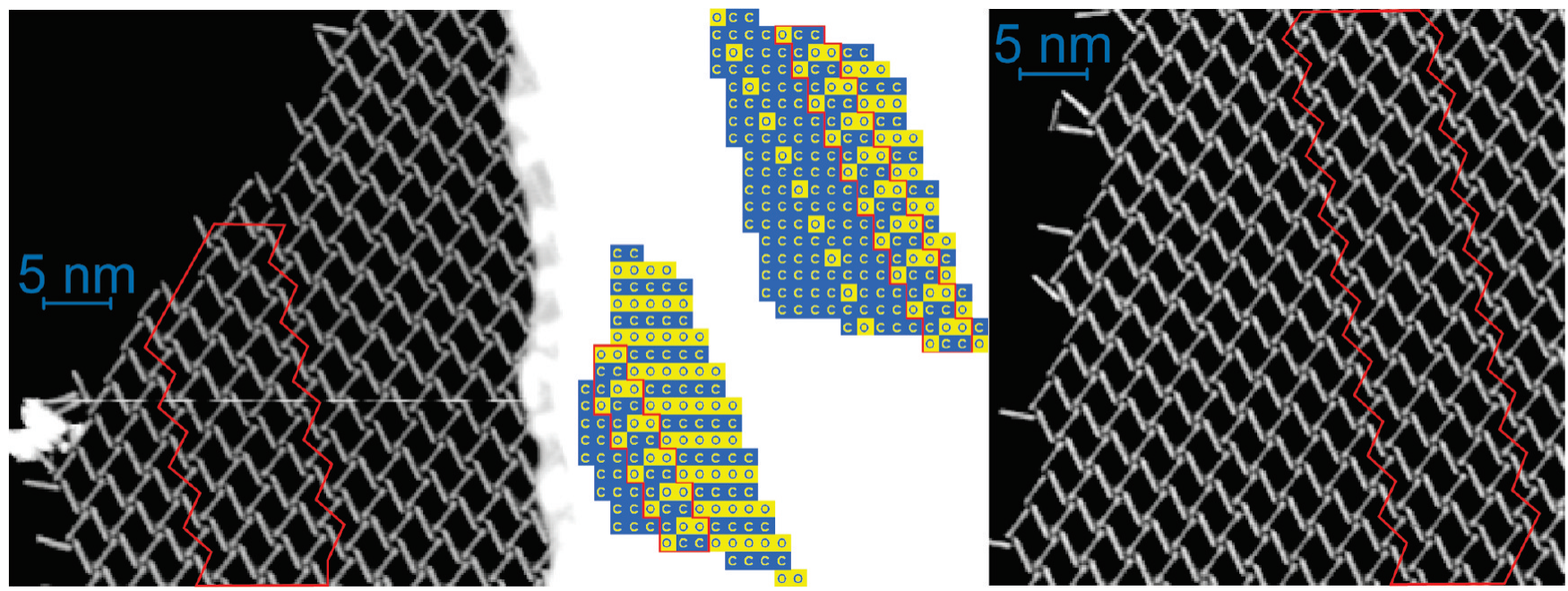

Figure 7. The distribution of open (o) and compact (c) nodes extracted from two network examples is encoded in the blue-yellow schemes. Both networks feature the same transition region surrounded by the red line in the schemes and in the STM data $\left(V_{\mathrm{B}}=0.65 \mathrm{~V}, I_{\mathrm{T}}=0.05 \mathrm{nA}\right)$.

\section{TABLE 1: Network Densities}

\begin{tabular}{lcccc}
\hline \multicolumn{1}{c}{ phase } & $\begin{array}{c}\text { unit cell } \\
\text { area/unit cells } \\
\text { of } \mathrm{Ag}(111)\end{array}$ & $\begin{array}{c}\text { number of } \\
\text { molecules/unit } \\
\text { cell }\end{array}$ & area/molecule & $\begin{array}{c}\text { of a ML } \\
\text { fraction }\end{array}$ \\
\hline kagomé & 427 & 6 & $5.14 \mathrm{~nm}^{2}$ & 0.406 \\
rectangular & 124 & 2 & $4.48 \mathrm{~nm}^{2}$ & 0.466 \\
rhombic & 218 & 4 & $3.94 \mathrm{~nm}^{2}$ & 0.530 \\
dense-packed & 231 & 8 & $2.09 \mathrm{~nm}^{2}$ & 1
\end{tabular}

groups near the hollow or bridge sites, which thus supports the epitaxy models given here. In addition, it was found that the cyano group is brought only slightly closer to the $\mathrm{Au}(111)$ surface. ${ }^{42}$ Even though the authors find a large amount of charge on the cyano group, which is attracted strongly by its image charge that appears in the metal, the tilt of the $\mathrm{CN}$ group with respect to the phenyl ring is negligible and justifies the assumption in our ball-and-stick models that the long molecular axis (from $\mathrm{N}$ to $\mathrm{N}$ ) remains straight for the adsorbed molecules.

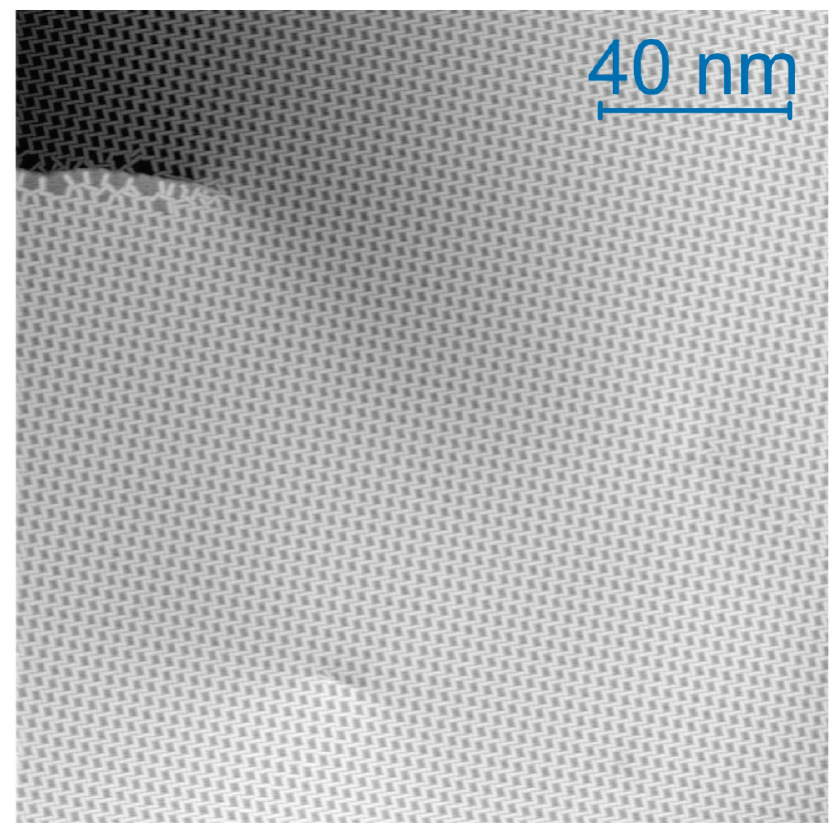

Figure 8. STM topography of the rectangular phase covering the surface around a screw dislocation of the $\operatorname{Ag}(111)$ surface. The network covers large areas with a small defect density, which originates mostly from substrate defects $\left(V_{\mathrm{B}}=1.90 \mathrm{~V}, I_{\mathrm{T}}=0.1 \mathrm{nA}\right)$.

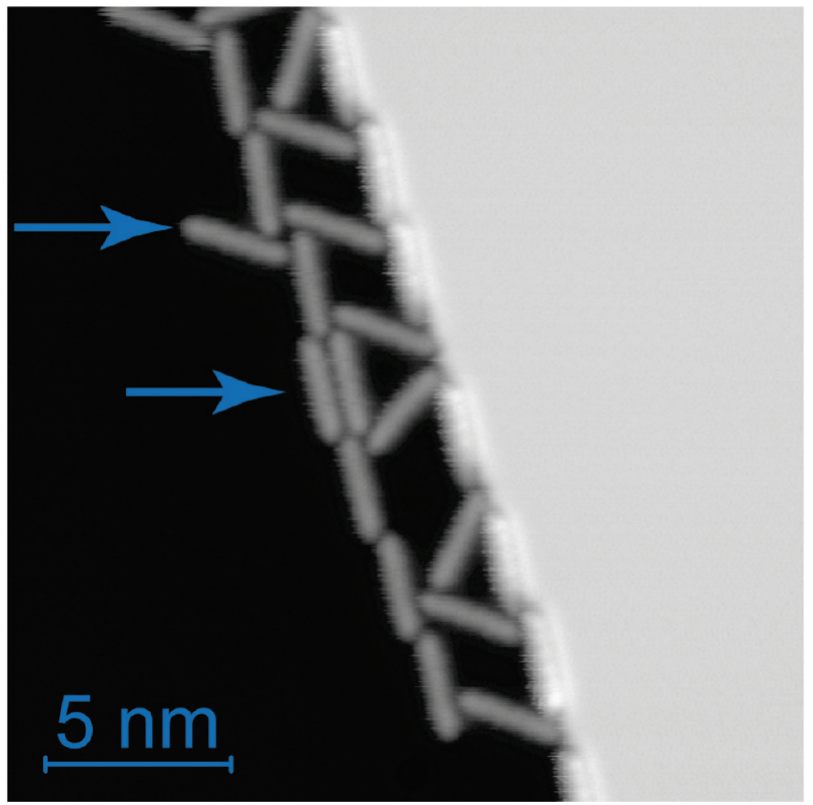

Figure 9. STM topography. Initial assembly takes place at step edges, as can be seen in this STM image of a sample with very little coverage (well below $0.1 \mathrm{MLs}$ ). Already in this stage, the basic binding motifs are present. The upper arrow points to a rotor motif, the lower one to a motif of the dense-packed network $\left(V_{\mathrm{B}}=1.48 \mathrm{~V}, I_{\mathrm{T}}=0.1 \mathrm{nA}\right)$.

Influence of Defects. Figure 8 shows the rectangular phase running around a screw dislocation of the $\operatorname{Ag}(111)$ surface. The rectangular network covers large areas and is highly regular. Defects in the substrate disturb the network merely locally but do not affect its overall regularity. In a series of consecutive images, we found that the domain size is limited by the terrace size, only. There is no phase fragmentation and domain sizes reach the micrometer range. This means elastic energy needs not be reduced $^{54}$ and there might be little strain in the commensurate phases.

As demonstrated in Figure 9, step edges are always found to be decorated with molecules even if there are no regular networks on the terraces to both sides of the step. The decoration occurs preferentially at the lower side of the step edges. A first row of molecules is bound parallel to the step edges, leaving some free places for a second row of molecules to bind to the step edges as well as to some molecules of the first row. This kind of boundary condition is likely to influence the assembly 
in the proximity of the steps, resulting in irregular networks on terraces with a width smaller than $20 \mathrm{~nm}$. Only if terraces are large enough, the influence of the steps can be overcome and regular networks form. Even in the near vicinity of a step edge, the preference of the molecule alignment for certain crystallographic directions and typical binding motifs is evident. The appearance of the 4-fold node motif (upper arrow), reminiscent of the porous networks, as well as the appearance of the parallel motif (lower arrow), reminiscent of the dense-packed phase, demonstrate that the regular networks are not only triggered by symmetry and commensurability with the substrate but also reside on the optimization of the bonding of the functional groups.

\section{Conclusions}

Submonolayer concentrations of sexiphenyl-dicarbonitrile molecules were self-assembled on $\operatorname{Ag}(111)$ and patterned the surface with three types of nanoporous networks with similar formation energy: a kagomé, rectangular, and rhombic network. All three networks are based on 4-fold nodal motifs and form large and regular domains. The motifs exhibit organizational 2D chirality; for each type, both enantiomers have been found. The commensurate networks could be modeled with the help of one construction principle: alignment of the long molecular axes along $\langle\overline{1} 10\rangle$ and $\langle\overline{1} 12\rangle$ combined with optimized $\mathrm{H} \cdots \mathrm{N}$ bonding in the 4-fold nodes. For coverages of sexiphenyldicarbonitrile close to ML saturation, networks transform into a dense-packed structure exhibiting a well-defined ordering of flat-lying molecules. The detailed insight into the registry of the porous organic adlayers with the underlying substrate provided by this work improves the understanding of the selfassembly of dicarbonitrile-polyphenyl networks. We have demonstrated that functional groups help to control the ordering of molecules with conjugated electron systems, which might increase the performance of future devices. ${ }^{38}$ As has been shown recently, ${ }^{55}$ nanoporous organic networks furthermore provide efficient means to tune electronic substrate properties.

\section{Experimental Section}

All experiments have been performed in an ultrahigh vacuum chamber housing standard sample preparation facilities at a background pressure of $3 \times 10^{-11}$ mbar offering clean and welldefined preparation conditions. We used a homemade beetletype scanning tunneling microscope (STM) operating at $8 \mathrm{~K}^{56}$ The $\operatorname{Ag}(111)$ substrate has been prepared by repeated cycles of $\mathrm{Ar}^{+}$sputtering and subsequent annealing at $480{ }^{\circ} \mathrm{C}$. The networks have been fabricated using organic molecular beam epitaxy of $\left[1,4^{\prime} ; 1^{\prime}, 1^{\prime \prime} ; 4^{\prime \prime}, 1^{\prime \prime \prime} ; 4^{\prime \prime \prime}, 1^{\prime \prime \prime \prime} ; 4^{\prime \prime \prime \prime}, 1^{\prime \prime \prime \prime \prime}\right]$ sexiphenyl-4, $4^{\prime \prime \prime \prime \prime}$ dicarbonitrile molecules (Chart 1) synthesized as described elsewhere. ${ }^{14}$ For evaporation, a Knudsen cell containing the molecules in a quartz crucible was used. The crucible was held at a temperature of $570 \mathrm{~K}$, leading to a deposition rate of one ML in 20 min where a ML is defined as a coverage such that the substrate surface is completely covered with a uniform, dense-packed layer with one molecule in $2.09 \mathrm{~nm}^{2}$ (Table 1). During deposition, the substrate temperature was constantly kept at 297 K. Subsequently, the sample was cooled down and transferred into the STM where data were recorded at $8 \mathrm{~K}$. The STM tip employed was an electrochemically etched tungsten wire (diameter: $0.2 \mathrm{~mm}$ ) cleaned inside the UHV chamber by $\mathrm{Ar}^{+}$self-sputtering. ${ }^{57}$

Acknowledgment. This work was supported by the European Science Foundation Collaborative Research Programme Fun-
SMARTs and by the DFG Cluster of Excellence Munich Center for Advanced Photonics.

Supporting Information Available: Additional STM data. This material is available free of charge via the Internet at http:// pubs.acs.org.

\section{References and Notes}

(1) Kudernac, T.; Lei, S. B.; Elemans, J. A. A. W.; De Feyter, S. Chem. Soc. Rev. 2009, 38, 402-421.

(2) Lin, N.; Stepanow, S.; Ruben, M.; Barth, J. V. Top. Curr. Chem. 2009, 287, 1-44. 203.

(3) Cicoira, F.; Santato, C.; Rosei, F. Top. Curr. Chem. 2009, 285,

(4) Barth, J. V. Annu. Rev. Phys. Chem. 2007, 58, 375-407.

(5) Griessl, S.; Lackinger, M.; Edelwirth, M.; Hietschold, M.; Heckl, W. M. Single Mol. 2002, 3, 25-31.

(6) Dmitriev, A.; Lin, N.; Weckesser, J.; Barth, J. V.; Kern, K. J. Phys. Chem. B 2002, 106, 6907-6912.

(7) Theobald, J. A.; Oxtoby, N. S.; Phillips, M. A.; Champness, N. R.; Beton, P. H. Nature 2003, 424, 1029-1031.

(8) Stöhr, M.; Wahl, M.; Galka, C. H.; Riehm, T.; Jung, T. A.; Gade, L. H. Angew. Chem., Int. Ed. 2005, 44, 7394-7398.

(9) Ruben, M.; Payer, D.; Landa, A.; Comisso, A.; Gattinoni, C.; Lin, N.; Collin, J. P.; Sauvage, J. P.; De Vita, A.; Kern, K. J. Am. Chem. Soc. 2006, 128, 15644-15651.

(10) Dmitriev, A.; Spillmann, H.; Lin, N.; Barth, J. V.; Kern, K. Angew. Chem., Int. Ed. 2003, 42, 2670-2673.

(11) Stepanow, S.; Lingenfelder, M.; Dmitriev, A.; Spillmann, H.; Delvigne, E.; Lin, N.; Deng, X.; Cai, C.; Barth, J. V.; Kern, K. Nat. Mater. 2004, 3, 229-233.

(12) Schlickum, U.; Decker, R.; Klappenberger, F.; Zoppellaro, G.; Klyatskaya, S.; Ruben, M.; Silanes, I.; Arnau, A.; Kern, K.; Brune, H.; Barth, J. V. Nano Lett. 2007, 7, 3813-3817.

(13) Stepanow, S.; Lin, N.; Barth, J. V. J. Phys.: Condens. Matter 2008, 20,184002 .

(14) Kühne, D.; Klappenberger, F.; Decker, R.; Schlickum, U.; Brune, H.; Klyatskaya, S.; Ruben, M.; Barth, J. V. J. Am. Chem. Soc. 2009, 131, 3881-3883.

(15) Schull, G.; Douillard, L.; Fiorini-Debuisschert, C.; Charra, F.; Mathevet, F.; Kreher, D.; Attias, A.-J. Nano Lett. 2006, 6, 1360-1363.

(16) Spillmann, H.; Kiebele, A.; Stöhr, M.; Jung, T. A.; Bonifazi, D.; Cheng, F.; Diederich, F. Adv. Mater. 2006, 18, 275-279.

(17) Mena-Osteritz, E.; Bäuerle, P. Adv. Mater. 2006, 18, 447-451.

(18) Tahara, K.; Furukawa, S.; Uji-i, H.; Uchino, T.; Ichikawa, T.; Zhang, J.; Mamdouh, W.; Sonoda, M.; De Schryver, F. C.; De Feyter, S.; Tobe, Y. J. Am. Chem. Soc. 2006, 128, 16613-16625.

(19) Zwaneveld, N. A. A.; Pawlak, R.; Abel, M.; Catalin, D.; Gigmes, D.; Bertin, D.; Porte, L. J. Am. Chem. Soc. 2008, 130, 6678-6679.

(20) Weigelt, S.; Bombis, C.; Busse, C.; Knudsen, M. M.; Gothelf, K. V.; Lægsgaard, E.; Besenbacher, F.; Linderoth, T. R. ACS Nano 2008, 2, 651660 .

(21) Mu, Z.; Shu, L.; Fuchs, H.; Mayor, M.; Chi, L. J. Am. Chem. Soc. 2008, 130, 10840-10841.

(22) Spillmann, H.; Dmitriev, A.; Lin, N.; Messina, P.; Barth, J. V.; Kern, K. J. Am. Chem. Soc. 2003, 125, 10725-10728.

(23) Stepanow, S.; Lin, N.; Barth, J. V.; Kern, K. J. Phys. Chem. B 2006, 110, 23472-23477.

(24) Vidal, F.; Delvigne, E.; Stepanow, S.; Lin, N.; Barth, J. V.; Kern, K. J. Am. Chem. Soc. 2005, 127, 10101-10106.

(25) Wintjes, N.; Bonifazi, D.; Cheng, F. Y.; Kiebele, A.; Stöhr, M.; Jung, T.; Spillmann, H.; Diederich, F. Angew. Chem., Int. Ed. 2007, 46, 4089-4092.

(26) Xiao, W.; Feng, X.; Ruffieux, P.; Gröning, O.; Müllen, K.; Fasel, R. J. Am. Chem. Soc. 2008, 130, 8910-8912.

(27) Schlickum, U.; Decker, R.; Klappenberger, F.; Zoppellaro, G.; Klyatskaya, S.; Auwärter, W.; Neppl, S.; Kern, K.; Brune, H.; Ruben, M.; Barth, J. V. J. Am. Chem. Soc. 2008, 130, 11778-11782.

(28) Fehlhammer, W. P.; Fritz, M. Chem. Rev. 1993, 93, 1243-1280. (29) Sleator, T.; Tycko, R. Phys. Rev. Lett. 1988, 60, 1418-1421.

(30) Kato, N. A.; Hara, M.; Sasabe, H.; Knoll, W. Nanotechnology 1996, 7, 122-127.

(31) Miller, J. S.; Epstein, A. J. Chem. Commun. 1998, 1319-1325.

(32) Miller, J. S. Inorg. Chem. 2000, 39, 4392-4408.

(33) Jain, R.; Kabir, K.; Gilroy, J. B.; Mitchell, K. A. R.; Wong, K.-c.; Hicks, R. G. Nature 2007, 445, 291-294.

(34) Yokoyama, T.; Yokoyama, S.; Kamikado, T.; Okuno, Y.; Mashiko, S. Nature 2001, 413, 619-621.

(35) Wintjes, N.; Hornung, J.; Lobo-Checa, J.; Voigt, T.; Samuely, T.; Thilgen, C.; Stöhr, M.; Diederich, F.; Jung, T. A. Chem.-Eur. J. 2008, 14, $5794-5802$. 
(36) Graupner, W.; Meghdadi, F.; Leising, G.; Lanzani, G.; Nisoli, M.; De Silvestri, S.; Fischer, W.; Stelzer, F. Phys. Rev. B 1997, 56, 1012810132.

(37) Grem, G.; Leditzky, G.; Ullrich, B.; Leising, G. Adv. Mater. 1992, 4, 36-37.

(38) Gundlach, D. J.; Lin, Y.-Y.; Jackson, T. N.; Schlom, D. G. Appl. Phys. Lett. 1997, 71, 3853-3855.

(39) Resel, R. Thin Solid Films 2003, 433, 1-11.

(40) Era, M.; Tsutsui, T.; Saito, S. Appl. Phys. Lett. 1995, 67, 24362438 .

(41) Leising, G.; Tasch, S.; Brandstätter, C.; Meghdadi, F.; Froyer, G.; Athouel, L. Adv. Mater. 1997, 9, 33-36.

(42) Okuno, Y.; Yokoyama, T.; Yokoyama, S.; Kamikado, T.; Mashiko, S. J. Am. Chem. Soc. 2002, 124, 7218-7225.

(43) Barlow, S. M.; Raval, R. Surf. Sci. Rep. 2003, 50, 201-341.

(44) Chen, W.; Zhang, H. L.; Huang, H.; Chen, L.; Wee, A. T. S. Appl. Phys. Lett. 2008, 92, 193301-3.

(45) Braun, K.-F.; Hla, S.-W. Nano Lett. 2005, 5, 73-76.

(46) Hla, S.-W.; Braun, K.-F.; Wassermann, B.; Rieder, K.-H. Phys. Rev. Lett. 2004, 93, 208302-4.

(47) Delugeard, Y.; Desuche, J.; Baudour, J. L. Acta Crystallogr., Sect. $B$ 1976, 32, 702-705.
(48) Ambrosch-Draxl, C.; Majewski, J. A.; Vogl, P.; Leising, G. Phys. Rev. B 1995, 51, 9668-9676.

(49) Guha, S.; Graupner, W.; Resel, R.; Chandrasekhar, M.; Chandrasekhar, H. R.; Glaser, R.; Leising, G. Phys. Rev. Lett. 1999, 82, 36253628 .

(50) Auwärter, W: Weber-Bargioni, A; Riemann, A; Schiffrin, A.; Gröning, O.; Fasel, R.; Barth, J. V. J. Chem. Phys. 2006, 124, 194708-6. (51) France, C. B.; Frame, F. A.; Parkinson, B. A. Langmuir 2006, 22, $7507-7511$.

(52) Vedmedenko, E. Y.; Mikuszeit, N. ChemPhysChem 2008, 9, 12221240

(53) France, C. B.; Schroeder, P. G.; Forsythe, J. C.; Parkinson, B. A. Langmuir 2003, 19, 1274-1281.

(54) Suo, Z.; Lu, W. J. Nanopart. Res. 2000, 2, 333-344.

(55) Klappenberger, F.; Kühne, D.; Krenner, W.; Silanes, I.; Arnau, A.; de Abajo, F. J. G.; Klyatskaya, S.; Ruben, M.; Barth, J. V. Nano Lett. [Online early access]. DOI: 10.1021/n1901700b. Published Online: June 17, 2009.

(56) Clair, S.; Pons, S.; Seitsonen, A. P.; Brune, H.; Kern, K.; Barth, J. V. J. Phys. Chem. B 2004, 108, 14585-14590.

(57) Albrektsen, O.; Salemink, H. W. M.; Mørch, K. A.; Thölen, A. R. J. Vac. Sci. Technol., B 1994, 12, 3187-3190.

JP9041217 